\title{
Kinematic Error Correction for Minimally Invasive Surgical Robots
}

\author{
Ryan A. Beasley and Robert D. Howe \\ Division of Engineering and Applied Sciences \\ Harvard University \\ Cambridge, USA \\ \{rbeasley, howe\}@deas.harvard.edu
}

\author{
Pierre E. Dupont \\ Department of Aerospace and Mechanical Engineering \\ Boston University \\ Boston, USA \\ pierre@bu.edu
}

\begin{abstract}
Robots are useful tools in minimally invasive surgery, providing benefits such as reduction in hand tremor, navigation, and workspace scaling. Unfortunately, minimally invasive configurations result in two likely sources of kinematic error: port displacement and instrument shaft flexion. For a quasistatic system, a measure is presented that relates the errors in the robot Jacobian to the angular difference between desired motions and actual motions. Simulations and experimental data demonstrate this measure for a laboratory system. One potential use for the presented measure is, for bounded errors, determining whether the system will monotonically converge for all initial and desired positions in the workspace. In addition, the measure is useful for path planning, determining less error-prone paths.
\end{abstract}

Keywords-surgical robot, image guidance, kinematic error

\section{INTRODUCTION}

Minimally invasive surgery (MIS) uses long instruments inserted through small ports in the patient's body. This technique greatly reduces patient trauma but increases surgical difficulty due to decreased dexterity and restriction of visual, tactile, and proprioceptive feedback. Robot assistance can help offset such complications by providing instrument localization, image guidance [1], navigational tools (e.g., virtual fixtures [2, 3]), dampening of hand tremor [4], and workspace scaling [5]. Unfortunately, laboratory and clinical trials demonstrate that errors in the robotic system result from uncertain kinematics, unmodeled dynamics, and unmodeled forces [2, 6]. Internal mammary artery takedown is an example task that exhibits such errors. To reach the surgical site near the chest wall, the instrument shaft applies significant torque to the port. The moments and forces created during the operation cause the patient's ribs to flex, the port to move, and the instrument shaft to bend. These kinematic errors impair positioning of the robot and cause deviations from the desired motions. Reducing the positioning precision reduces the navigational benefits of the robot. In the worst case, a slave robot may move in directions other than those of the master robot, decreasing patient safety.

Much work has been done on reducing kinematic error. Offline calibration calculates exact kinematic parameters, but does not account for online errors due to interactions with unstructured environments $[7,8]$. Estimating the bending of the instrument shaft is problematic due to unmodeled forces at the port and within the patient. Research into flexible robotics has focused on dynamic flexibility [9, 10]. Such research assumes known static kinematics, which is not the case when there is static deformation against the patient. Measuring the position of the tip of the instrument shaft using cameras or magnetic position trackers is another approach. Such measurements eliminate the problem of errors in the forward kinematics, but do not directly solve the problem of errors in the inverse kinematics. One method for using that position information to reduce the effects of errors in inverse kinematics is by online estimation of the local Jacobian $[11,12]$. Unfortunately, significant motion in a single direction can lead to errors in these estimates. Another method of using position measurements to correct for kinematic errors is to use a controller based on the nominal Jacobian. Cheah, et al., derive a relationship between error in a transpose Jacobian controller and the controller gains that result in asymptotic convergence to the commanded position. While that result is useful, for surgical robotics it is important to have more information, particularly concerning motion of the robot before convergence.

For robots using an inverse Jacobian controller, this paper presents a measure of the positioning error in the controller based on the error in the Jacobian. That measure can be used to determine whether the system is monotonically convergent, and to estimate the maximum angular difference between desired motions and actual motions as produced by the controller. The maximum angular difference can then be used to determine how closely the system would follow commanded motions. Simulations and experimental data are used to illustrate the measure for a laboratory robot system. Practical uses for the measure are then discussed such as predicting monotonic convergence, path planning, and robot design.

\section{ANALYSIS OF CONTROLLER}

An ideal robotic system moves to the commanded position and does not stray from the most direct path to that position. The first trait translates to asymptotic convergence and the second trait is related to monotonic convergence. For design and safety reasons, it is important to have a measure of the effect that errors in the controller have on these properties of the system. The measure developed here uses a quasi-static assumption to calculate both the Cartesian error length after a

This work was supported by the National Science Foundation under grant EEC-9731748. 


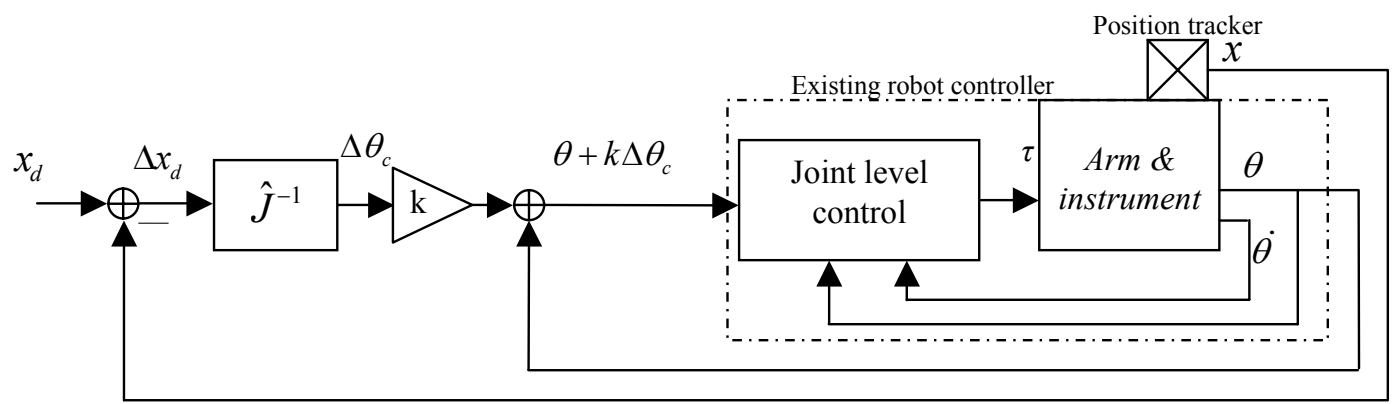

Figure 1. Block diagram of controller.

motion and the angle between desired and actual motions. Possible applications in control of orientation have been left for future work.

\section{A. Controller and Assumptions}

An important use of surgical robots is for image-guided surgery. The surgeon watches images from cameras and preoperative scans while manipulating the master robot. The motions of the master are copied by the slave robot, which holds a surgical instrument. In MIS, instruments have long shafts that pass through a small port in the patient's body.

Unfortunately, kinematic errors in the slave robot result in incorrect image guidance, navigational aids (e.g., virtual fixtures), and robot motions. Directly measuring the robot position with cameras or a magnetic tracker can fix the problems with image guidance, but the actual motions of the robot would still not match the commanded motions.

Focusing on MIS robots influences the choice of feedback controllers. Many surgical robots have a built-in joint-level controller. That controller is running with a high servo rate and has critically damped or over-damped behavior, with properties set at initial design. Additionally, the joint-level controller has regulatory approval and has been used in thousands of surgical cases, factors that inhibit modifications. Often the kinematics for the master and slave robots are dissimilar, so an inverse Jacobian controller is practical for the outer loop controller. The inverse Jacobian controller requires the current position of the robot, which can be measured via camera or magnetic position sensor. Combining these into one system (Fig. 1), the desired position for the robot can be set and the robot will move to that position, barring kinematic errors.

While contacts between the instrument shaft and the environment can introduce errors, these contacts have the benefit of reducing the effects of system dynamics. The contacts increase the damping of the system, leading to a quasistatic situation. Critically damped joint-level control also leads to a quasi-static situation due to its minimum settling time and no overshoot. With these features, a quasi-static assumption holds as long as the commanded angular changes are small enough to be completed before the next update of the inverse Jacobian controller. To satisfy this constraint, the motion scaling gain of the controller is decreased until the system behaves in a quasi-static manner.

\section{B. Derivation of Error Measures}

For an inverse Jacobian controller with an imperfect Jacobian, the commanded change in joint angles, $\Delta \theta_{c} \in \mathbb{R}^{n}$ where $n$ is the degrees of freedom for the robot, is calculated as

$$
\Delta \theta_{c}=k \hat{J}^{-1}\left(x_{d}-x\right)=k \hat{J}^{-1} \Delta x_{d},
$$

where $x \in \mathbb{R}^{3}$ is the current Cartesian position, $x_{d} \in \mathbb{R}^{3}$ is the desired Cartesian position, $\Delta x_{d} \in \mathbb{R}^{3}$ is the desired motion in Cartesian space, $\hat{J}$ is an estimate of $J$, the true system Jacobian, and $k$ is a motion scaling gain. For infinitesimal steps and an ideal quasi-static system, the resulting motion is

$$
\Delta x=J \Delta \theta_{c}=k J \hat{J}^{-1} \Delta x_{d} .
$$

Therefore the new position after completing the motion is

$$
x_{\text {new }}=\Delta x+x=k J \hat{J}^{-1} \Delta x_{d}+x,
$$

and the error after completing the motion is

$$
\Delta x_{\text {new }}=x_{d}-x_{\text {new }}=x_{d}-x-k J \hat{J}^{-1} \Delta x_{d} .
$$

A mapping, $G(\theta, k)$, is derived from $\Delta x_{d}$ to $\Delta x_{\text {new }}$.

$$
\Delta x_{\text {new }}=G(\theta, k) \Delta x_{d}=\left(I-k J \hat{J}^{-1}\right) \Delta x_{d} .
$$

To analyze convergence, the induced Euclidean norm of $G(\theta, k)$ can be defined in the standard way.

$$
\|G(\theta, k)\|_{i 2}=\max _{\Delta x_{d} \neq 0} \frac{\left\|G \Delta x_{d}\right\|_{2}}{\left\|\Delta x_{d}\right\|_{2}}=\bar{\sigma}(G),
$$

where $\bar{\sigma}(G)$ denotes the maximum singular value of $G$. If $\|G(\theta, k)\|_{i 2}<1, \forall \theta$, the Cartesian error length is always smaller after a step than before the step. Therefore, the Cartesian error length monotonically decreases to zero with subsequent steps, making the controller monotonically and asymptotically convergent. Let $v_{\sigma}$ be the right singular vector that corresponds to the maximum singular value, $\bar{\sigma}(G)$. Note that $v_{\sigma}$ is the desired direction that results in the largest $\left\|\Delta x_{\text {new }}\right\|$. Also note, neither $\|G(\theta, k)\|_{i 2}$ nor $v_{\sigma}$ have any application to the orientation of the robot.

While $\|G(\theta, k)\|_{i 2}$ is useful, a potentially more important measure is the angle, $\phi$, between the desired motion, $\Delta x_{d}$, and the actual motion, $\Delta x$ (Fig. 2). If $|\phi|$ is small for all possible motions across the workspace, then for a teleoperated system 


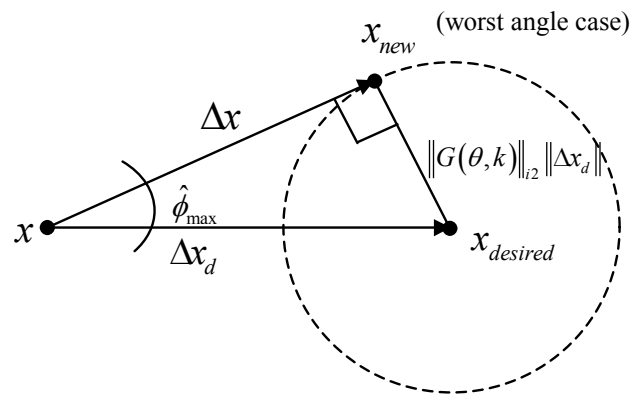

Figure 2. Geometry of $\hat{\phi}_{\max }$.

the slave robot will accurately follow the motions of the master. Let $\left|\phi_{\max }(\theta)\right|$ be the maximum absolute value of $\phi$ over all desired motions, for a specific joint configuration, $\theta$. From linear algebra,

$$
G v_{i}=\sigma_{i} u_{i}
$$

where $v_{i}$ are the right singular vectors of $G, u_{i}$ are the left singular vectors of $G$, and $\sigma_{i}$ are the corresponding singular values. So pairs of input and output vectors can be defined where the input vectors, $\bar{v}$, are unit length,

$$
\left(\bar{v}=\sum_{i=1}^{m} \alpha_{i} v_{i}, \sum_{i=1}^{m} \alpha_{i}^{2}=1\right) \overrightarrow{\vec{G}}\left(\bar{u}=\sum_{i=1}^{m} \sigma_{i} \alpha_{i} u_{i}\right),
$$

where $m=\operatorname{dim}(x)$. Then

$$
\left|\phi_{\max }(\theta)\right|=\max _{\bar{v}}\left\{\cos ^{-1}\left(\frac{\bar{v}^{T}(\bar{v}-\bar{u})}{\|\bar{v}-\bar{u}\|_{2}}\right)\right\} .
$$

Equation (9) calculates the exact value of $\left|\phi_{\max }(\theta)\right|$, but requires searching over a sphere of radius 1, invoking computational requirements tolerable for offline calculations but that may be too great for real-time use. Instead, an upper bound on $\left\{\phi_{\max }(\theta) \mid\right.$ can be determined geometrically from

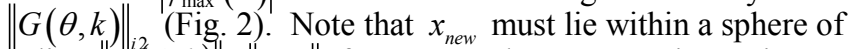
radius $\|G(\theta, k)\| \Delta x_{d} \|$ from $x_{d}$. The conservative estimate, $\hat{\phi}_{\max }(\theta, k)$, of $\phi_{\max }(\theta)$ is constructed by assuming the actual motion has the largest possible $|\phi|$. Then the actual motion is tangential to the surface of the sphere about $x_{d}$, putting a right angle between the actual motion and the radius of the sphere. The resulting right triangle has the desired motion as its hypotenuse. An upper bound on $\phi_{\max }(\theta)$ is therefore

$$
\begin{aligned}
\hat{\phi}_{\max }(\theta, k) & =\sin ^{-1}\left(\frac{\|G(\theta, k)\|_{i 2}\left\|\Delta x_{d}\right\|}{\left\|\Delta x_{d}\right\|}\right) \\
& =\left\{\begin{array}{cc}
\sin ^{-1}(\bar{\sigma}(G)) & , \bar{\sigma}(G) \leq 1 \\
\pi & , \bar{\sigma}(G)>1 .
\end{array}\right.
\end{aligned}
$$

Let $p_{\phi}$ be the direction of desired motion that results in $\left|\phi_{\max }\right|$. Note that there is no simple relationship between $p_{\phi}$ and $v_{\sigma} . \quad v_{\sigma}$ is derived from $\|G(\theta, k)\|_{i 2}$, a measure of the maximum length of positional error, unlike $\left|\phi_{\max }\right|$, which directly measures the angular error.

\section{EXAMPLES}

To demonstrate the various measures and variables, a simplified MIS robot system was simulated quasi-statically using the controller in Fig. 1. Kinematic errors due to displacement of the instrument port were introduced, and $\left|\phi_{\max }\right|,\|G(\theta, k)\|_{i 2}, \hat{\phi}_{\max }, p_{\phi}$, and $v_{\sigma}$ were calculated across the workspace of the system. This source of error was chosen for its ease of implementation and straightforward implications. Additionally, the experimental setup (Fig. 3), using the controller in Fig. 1, generated trajectories from various initial and desired positions in the presence of kinematic errors, and these trajectories were compared with trajectories calculated via simulation.

\section{A. Computer Simulation}

The simulation consisted of a planar two-link robot attached to an instrument shaft. Fig. 4 displays the workspace and configuration for both the simulation and experiment. The workspace of the robot's wrist is broken into two classifications depending on whether the instrument shaft could reach the port with the robot at that position. The links were each $210 \mathrm{~mm}$ and the instrument shaft was $320 \mathrm{~mm}$. The home position was with the first link horizontal (parallel to the $z$-axis) and the second link vertical (parallel to the $y$-axis). The origin was at the wrist of the robot in its home position. The instrument shaft passed through a port that was placed at $(y=0$, $z=160)$. We assume that the first joint is constrained to angular values between 1.2 and -1.9 radians as measured from the home position. The second joint is constrained to angular values between 1.0 and -0.7 radians as measured from the home position. Additionally, the joints are constrained such that the sum of the angles for both joints is between 2.8 and 0.3 radians. These joint constraints are similar to the physical constraints of the robot used in the experimental setup, described in the next section.

For each point in the tip workspace, we calculate the error metrics with a port positioning error of $+20 \mathrm{~mm}$ in $y$ and +10 $\mathrm{mm}$ in $z$, and assuming quasi-static steps (Fig. 5). This port error is somewhat larger than measured in our animal trials (Zeus surgical robot system, Computer Motion Inc., Goleta, California), but serves to illustrate behavior of the error metrics. The circle is the location of the port and the ' $x$ ' is the estimated location of the port used by the controller. Fig. 5a plots the induced Euclidean norm, $\|G(\theta, 1)\|_{i 2} \cdot\|G(\theta, 1)\|_{i 2} \geq 1$ for a large portion of the workspace; therefore the system is not guaranteed to be monotonically convergent over the entire workspace. However, $\|G(\theta, k)\|_{i 2} \leq 0.5$ in the middle of the workspace, so small desired motions there should be monotonically convergent. In general, as the controller gain, $k$, decreases from unit gain to zero, the commanded motions shrink in length and so $\|G(\theta, k)\|_{i 2}$ constricts to values closer to one. As $k$ increases from unit gain, $\|G(\theta, k)\|_{i 2}$ increases proportionally, due to the larger motions. The regions signified by the points $L, M$, and $N$ in Fig. 5 were used in the experimental tests displayed in Fig. 8. 


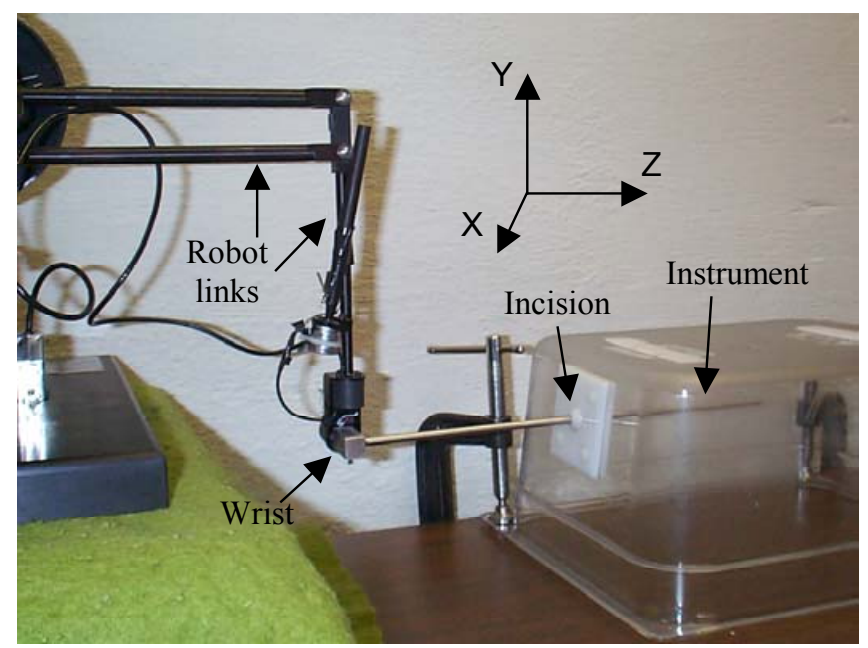

Figure 3. Experimental setup with axes.

Fig. $5 \mathrm{~b}$ shows the estimate, $\hat{\phi}_{\max }(\theta, k)$ with unit gain. The effects of changing $k$ on $\hat{\phi}_{\max }$ are to drive it to either 90 degrees for a $k$ decreasing to zero or 180 degrees for a $k$ increasing to infinity, as required by (10) and the effects of $k$ on $\|G(\theta, k)\|$. Fig. $5 \mathrm{c}$ shows the maximum absolute angular error, $\left|\phi_{\max }(\theta)\right|$. This plot is unaffected by changes in the controller's motion scaling gain, $k$. The latter two plots demonstrate that $\hat{\phi}_{\max }$ is a conservative estimate of $\left|\phi_{\max }\right|$. In fact, the contour line at the right of the workspace, beyond which $\hat{\phi}_{\max }$ is greater than 135 degrees, approximately corresponds to where $\left|\phi_{\max }\right|$ is greater than 45 degrees. Examining $\left|\phi_{\max }\right|$, the actual motion is within 90 degrees of the desired motion for most of the workspace. In all three plots, larger values occurred when either the robot or the shaft tip were near the port position, locations at which the effects of the port's position error on the controller are greatest.

Fig. $6 \mathrm{a}$ is a vector plot of $v_{\sigma}$ across the workspace, displaying the directions of desired motion that maximized $\left\|\Delta x_{n e w}\right\|$. This plot shows the desired motion directions that result in the largest Cartesian error lengths after the actual motions. Of interest are the locations where nearby vector lines are perpendicular to each other, because at those locations small changes of position cause large changes in $v$, such as in the lower left corner and around the port. Except in the lower left corner, the $v_{\sigma}$ for positions along the edge of the workspace are all approximately perpendicular to the boundary of the workspace. Fig. 6b contains the same line segments scaled by $\|G(\theta, k)\|_{i 2}$, which shows that even though $v$ is behaving interestingly in the center of the workspace, the errors there are not significant.

Fig. $6 \mathrm{c}$ is a vector plot of $p_{\phi}$, the directions of desired motions that result in $\left|\phi_{\max }(\theta)\right|$. These vectors are similar to the ones in Fig. 6a, but the differences are rather noticeable in the bottom left, and along the edge of the workspace. Also, $p_{\phi}$ seems to change more smoothly than $v_{\sigma}$, around the left-center of the workspace. Fig. 6d is the same plot scaled by $\left|\phi_{\max }(\theta)\right|$. Comparing Fig. 6d to Fig. 6b emphasizes the difference between $v$ and $p_{\phi}$ where errors in motion are largest, near the workspace boundary furthest from the port.

To show the effect of different error directions in the port estimate, Fig. 7 contains simulation data for various port

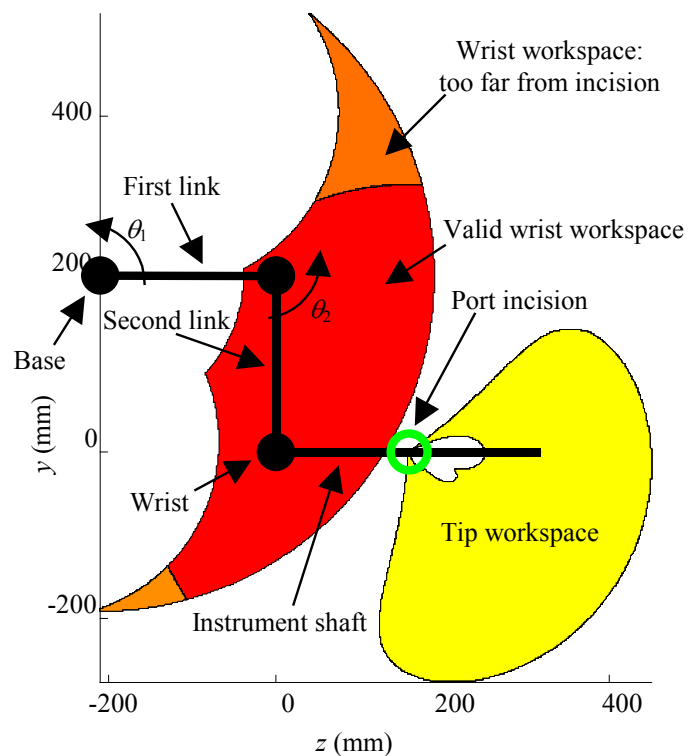

Figure 4. Diagram of wrist and tip workspaces, with links and shaft as lines and port as a circle.

positioning errors. The errors are $10 \mathrm{~mm}$ in the $+z,-z,+y$ and $y$ directions, respectively. Plots of $\left|\phi_{\max }(\theta)\right|$ and $\hat{\phi}_{\max }(\theta, 1)$ are shown. $\hat{\phi}_{\max }$ is again shown to be a conservative estimate of $\left|\phi_{\max }\right|$. Port position errors in the $z$-direction cause greater error at the bottom of the workspace, whereas port errors in the $y$ direction cause more error on the right side of the workspace. Looking more closely, positive $y$ errors in the port position cause the high error region to shift downward compared to negative $y$ errors in the port position. The trend is positions along the line of port error do not have errors in motion.

\section{B. Physical Experiment}

The experimental setup (Fig. 3) incorporated a Phantom robot (model 1.5, Sensable Technologies, Inc., Woburn, MA). For this setup, the roll joint was removed from the wrist and all positions were kept in the $y-z$ plane by actively driving the base joint to a set value. Limiting motion to the vertical plane allows easy visualization of results. The home position of the Phantom is with the first link horizontal and the second link vertical. The origin of graph axes is at the position of the tip of the robot in its home position. The controller in Fig. 1 was used to control the robot. The joint level controller was PID with the same update rate as the inverse Jacobian control. The various gains were chosen for a slow, over-damped motion to any position within the workspace.

A hollow cylinder $320 \mathrm{~mm}$ in length and made of stainless steel was attached to the end of the Phantom to simulate a surgical instrument. The Jacobian in the inverse Jacobian controller relates the change in the position of the tip of this shaft to the change in the robot joint angles. This shaft passed through a model of a port constraint. The port constraint consisted of a low friction acetyl ball joint. The ball freely rotated but could not translate. A cylindrical hole in the ball allowed the shaft to pass through. For this work, the port constraint was placed halfway along the shaft in the $z$ direction, 

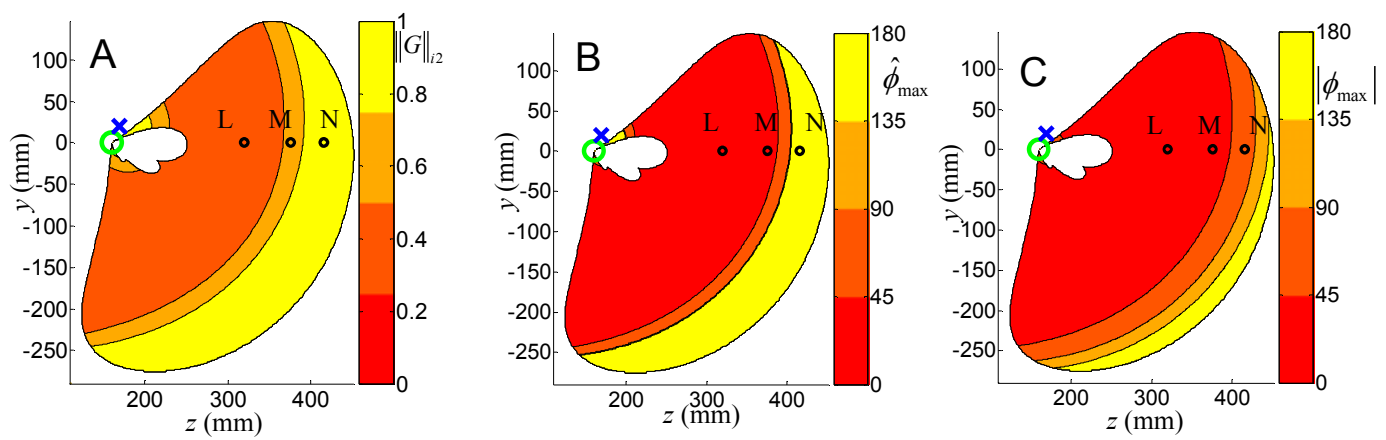

Figure 5. (A) $\|G\|_{i 2}$, (B) $\hat{\phi}_{\max }$ (degrees), and (C) $\left|\phi_{\max }\right|$ (degrees) for port positioning error of $(20,10)$ $\mathrm{mm}$. The circle shows the true port location. The ' $\mathrm{X}$ ' is the estimated port location.
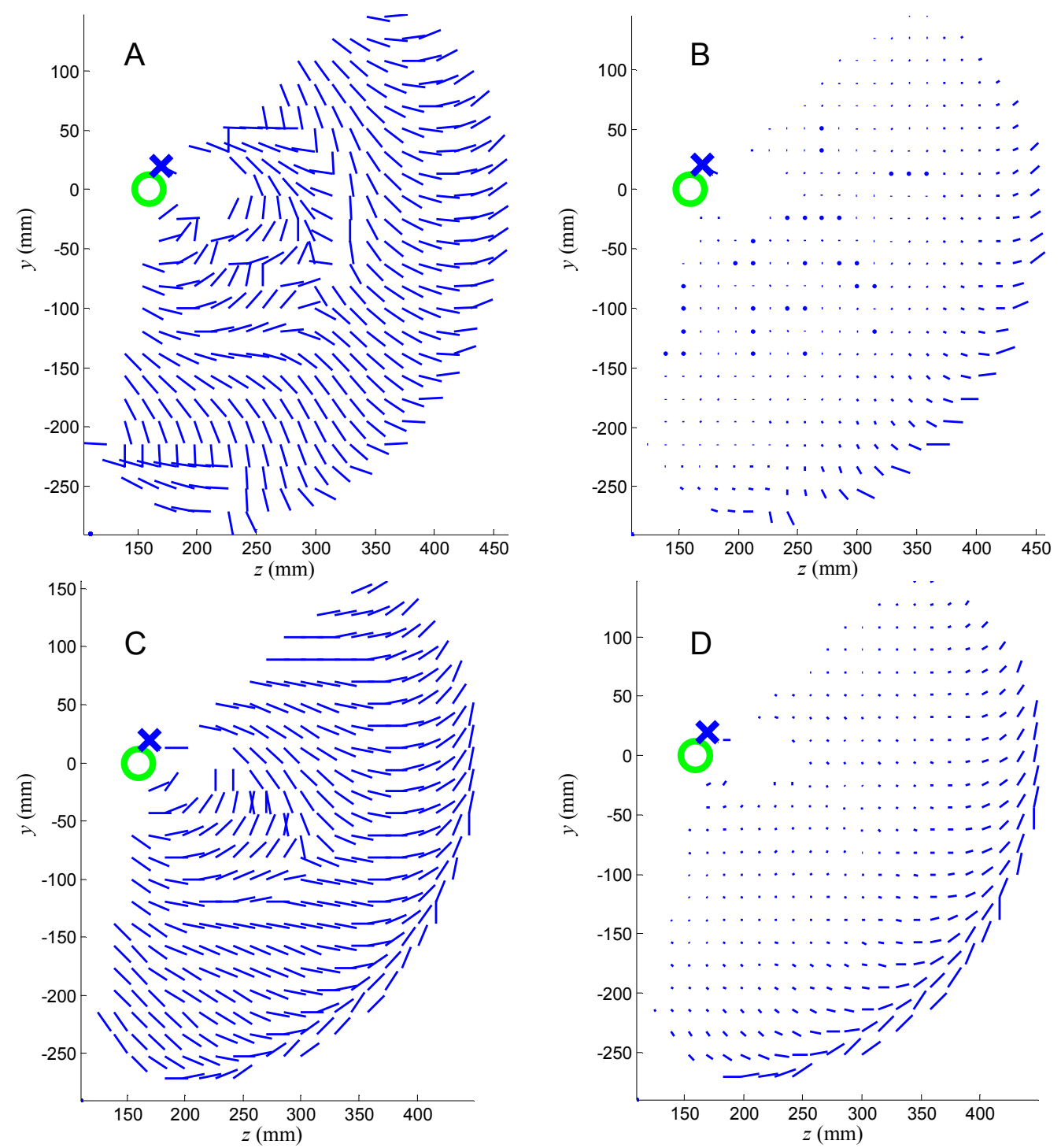

FIGURE 6. (A) Direction of desired motions that result in maximum Cartesian error length, $v_{\sigma}$; (B) $v_{\sigma}$ scaled by $\|G\|_{i 2}$; (C) Direction of desired motions that result in maximum absolute angular error, $p_{\phi}$; (D) $p_{\phi}$ scaled by $\left|\phi_{\max }\right|$. The circle shows the true incision location. The ' $x$ ' is the estimated incision location. 
at position $(y=0 \mathrm{~mm}, z=160 \mathrm{~mm})$. Fig. 4 shows the workspace of the robot's wrist and the workspace of the tip of the shaft. The controller used an estimated port position of $(20,170)$. This port error was the only kinematic error in the experiment. Since the true port position was known, the instrument tip position ( $x$ in Fig. 1) was calculated using forward kinematics.

For the experiment, initial tip positions were placed at $(y, z)$ : $(0,320),(0,375)$, and $(0,415)$, labeled $L-N$ in Fig. 5 . Those positions were chosen to lie in areas of increasing error. Eight stop positions were then chosen for each start position at distances of $\pm 20 \mathrm{~mm}$ along the $y$-axis, $z$-axis and diagonal directions. Experimental position data were gathered as the system moved from each start position to the corresponding eight desired positions. Fig. 8 contains plots of the experimental trajectories described for the desired points $L, M$, and $N$, respectively. Simulated paths overlay the plots in dashed lines, calculated as quasi-static steps with a controller gain of 0.01 . Though none of these results show the robot moving away from the desired position before converging, such a path is possible. The simulated path for the desired position at $(435,0)$ actually does move away from the desired position just before converging.

\section{DISCUSSION}

Robots can be useful tools in minimally invasive surgery, increasing situational awareness, reducing hand tremor, and providing tools to reduce the surgeon's mental workload. Unfortunately, experience shows that port motion and flexing of the instrument shaft can result in localization and motion errors, mitigating the helpfulness of the robot. Such errors can reduce or eliminate the usefulness of navigational aids and image guidance, as well as cause the robot to move the instrument in undesirable directions.

A measure, $\hat{\phi}_{\max }$, was proposed for minimally invasive surgical robots with inverse Jacobian controllers. Given the true Jacobian and the estimated Jacobian, $\hat{\phi}_{\max }$ conservatively estimates the maximum absolute angle, $\left|\phi_{\max }\right|$, between desired motion and actual motion. This measure assumes a quasi-static system, an assumption justified by low gain on the inverse Jacobian controller, a high-speed joint-level controller, and the presence of damping.

The simulation demonstrates the measures of the motion error across the workspace. Comparing Fig. $5 \mathrm{~b}$ and $5 \mathrm{c}, \hat{\phi}_{\max }$ is larger than $\left|\phi_{\max }\right|$ at all positions, behaving as a conservative estimate. The contours of $\hat{\phi}_{\max }$ are much steeper than those of $\left|\phi_{\max }\right|$. This results in $\hat{\phi}_{\max }$ closely estimating $\left|\phi_{\max }\right|$ around the middle of the workspace, but being quite large at positions close to, or far from, the port.

In the purest sense, $\hat{\phi}_{\max }$ and $\|G(\theta, k)\|_{i 2}$ are not practically usable metrics since they require knowledge of the true Jacobian of the system. One practical use does exist when the error sources on the kinematics are bounded. In this case, calculating $\|G(\theta, k)\|_{i 2}$ for the worst cases shows whether the robot will converge with a monotonically decreasing error length for all possible cases, and allows the observation of subsets of the workspace where the behavior of the system is most desirable. Similarly, the measure can be used as a design
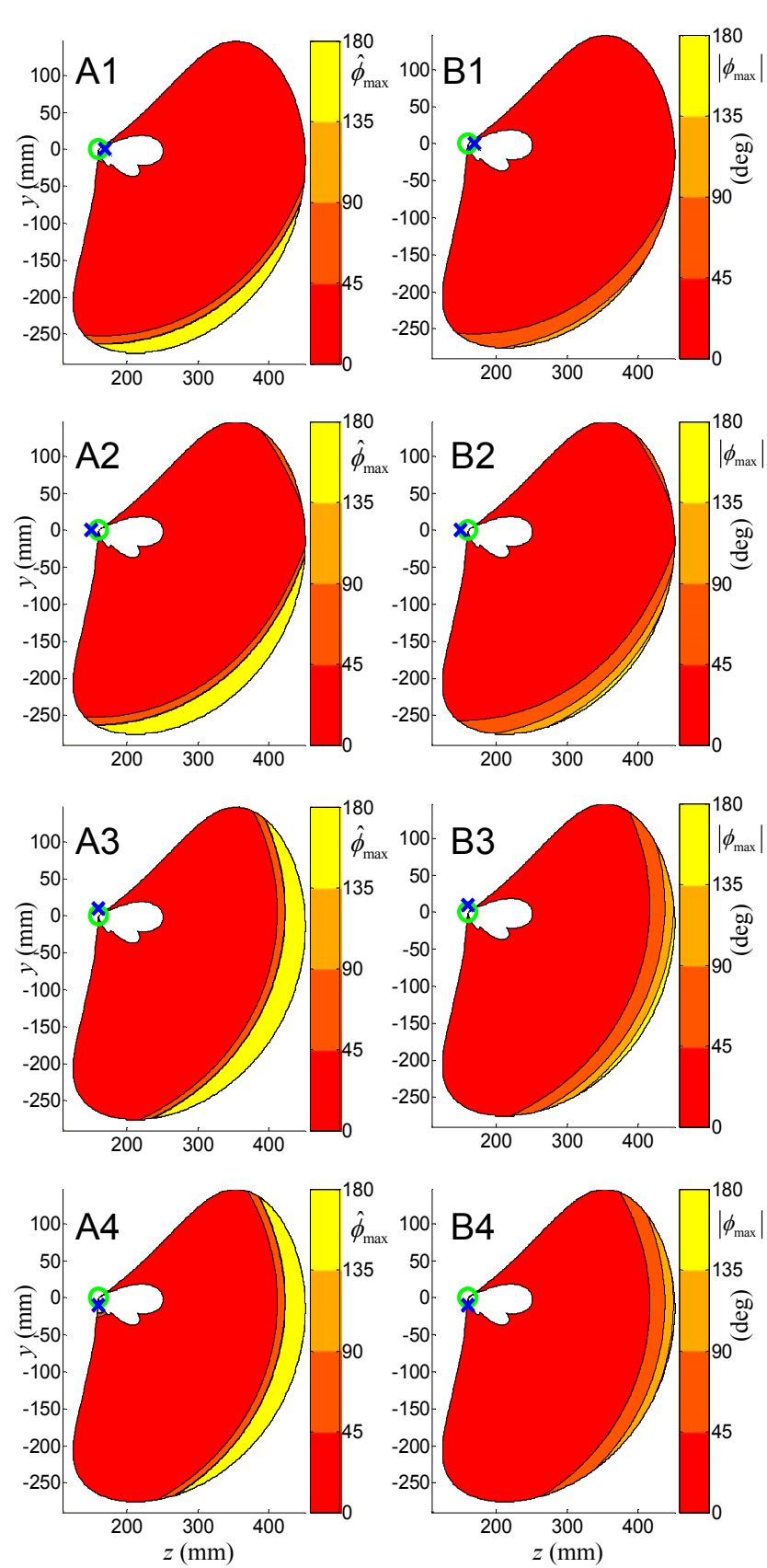

Figure 7. (A) $\hat{\phi}_{\max }$ (degrees). (B) $\left|\phi_{\max }\right|$ (degrees). Each with $10 \mathrm{~mm}$ port positioning error in a different direction: (1) $+z$. (2) $-z$. (3) $+y$. (4) $-y$. A circle shows the true incision location. An ' $\mathrm{X}$ ' shows the estimated incision location.

tool to determine the necessary tolerances of various system components. Using the system in this paper as an example, if positions along $y=0$ are the most important, then Fig. 7 shows that errors in the $y$ position of the port are less tolerable than errors in the $z$ position.

Another practical result lies in using angular errors to predict error sources. Using an external position measuring system to track the tip position, a plot of the measured errors in angle, $\phi$, throughout the workspace could be composed. By comparing those measurements to the various plots of 


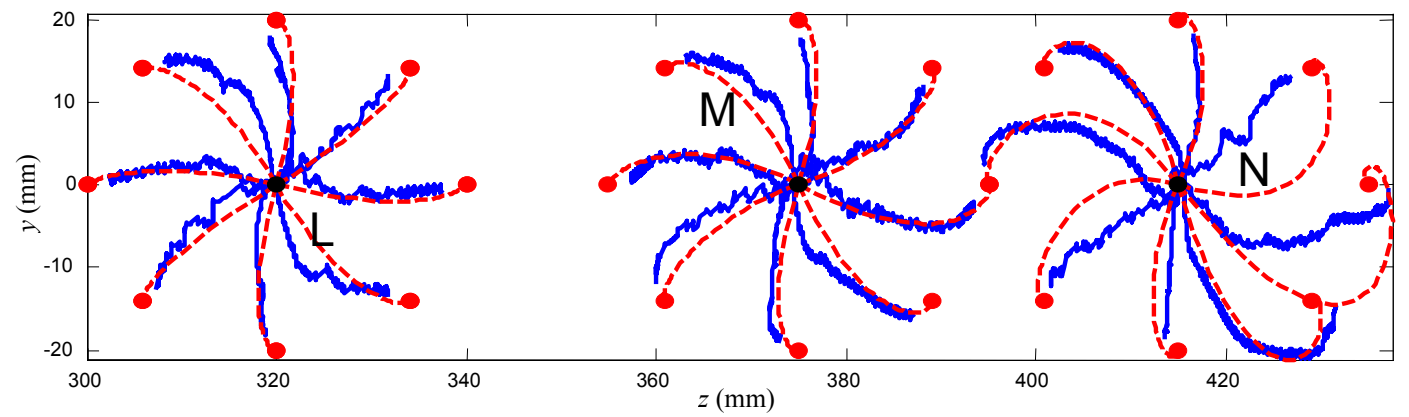

Figure 8. Trajectories followed in experiment (solid lines) and simulation (dashed lines), to the desired positions (red dots) each 20 $\mathrm{mm}$ away from the corresponding start positions (black dots) at $(\mathrm{L}):(320,0),(\mathrm{M}):(375,0),(\mathrm{N}):(415,0)$.

$\phi_{\max }(\theta) \mid$ generated by assuming different sources of error, similarities could suggest which sources of error are significant.

Online calculation of $\hat{\phi}_{\max }(\theta, k)$ could use a priori knowledge to enhance online estimation of the Jacobian. By assuming bounds for sources of significant error, worst-case Jacobians can be calculated for the errors at each of those bounds. The Jacobian can then be estimated during online motions, allowing calculations of $\hat{\phi}_{\max }(\theta, k)$ between the estimated Jacobian and each of the worst-case Jacobians. The maximum of those angles provides an upper bound on the actual angular error.

The desired motions, $v_{\sigma}$, plotted in Fig. $6 \mathrm{a}$, are similar to the desired motions, $p_{\phi}$, in Fig. 6c. Therefore, the desired motions that result in the largest length of Cartesian error for a unit controller gain are similar to the motions that actually result in the largest angular error. The differences occur wherever changing the motion scaling gain of the inverse Jacobian controller could decrease the Cartesian error.

Path planning is another practical use for this work. If the likely sources of error are known, $v_{\sigma}$ can easily be calculated for those error sources. A path planner could then trade off between indirect and direct paths to the desired final position, where the indirect paths may have less error than the direct paths.

The experimental paths in Fig. 8 approximately follow the simulated paths, providing some experimental validation for the simulation. The most obvious differences are for the start position at $(415,0)$, the start position closest to the edge of the workspace. For this start position, three of the experimental paths do not closely follow the simulated paths, those for the desired positions at $(429,14),(401,-14)$, and $(435,0)$. Another difference between experimental and simulated paths in Fig. 8 is that the angular error between desired motion and actual motion is sometimes greater for the experimental paths than for the simulated paths. The combination of three sources of error could explain these discrepancies. First, friction at the port constraint impedes motion anisotropically based on velocity. Second, lack of gravity compensation in the joint-level control results in unmodeled forces on the system that vary over the workspace. Third, dynamic effects could influence the experimental system, though this seems unlikely since the controller gains were set for very slow motion.
Future studies will apply this work to shaft flexion as a source of kinematic error. Subsequently, the two sources of error, port motion and instrument flexion, will be combined. The result will be bounds on those error sources such that the system will still converge with a monotonically decreasing positional error length. An additional possibility is to investigate the relationship between the measures presented in this paper and the orientation of the robot by using the full Jacobian instead of just the position portion of the Jacobian.

\section{REFERENCES}

[1] T. M. Peters, "Image-guided surgery: from X-rays to virtual reality," Computer Methods in Biomechanics and Biomedical Engineering, vol. 4, pp. 27-57, 2000.

[2] S. S. Park, R. D. Howe, and D. F. Torchiana, "Virtual fixtures for robotassisted minimally-invasive cardiac surgery," Medical Image Computing and Computer-Assisted Intervention 2001, W.J. Niessen and M.A. Viergever, Eds. Berlin: Springer-Verlag, Berlin, 2001, pp. 1419-1420.

[3] J. J. Abbott and A. M. Okamura, "Virtual fixture architectures for telemanipulation," IEEE Intl. Conf. on Robotics and Automation, pp. 2798-2805, 2003.

[4] L. Panait, C. Doarn, and R. Merrell, "Applications of robotics in surgery," Chirurgia Bucuresti, vol. 97, no. 6, pp. 549-55, 2002.

[5] G. Guthart and K. Salisbury, Jr., "The IntuitiveTM telesurgery system: overview and application," IEEE Intl. Conf. on Robotics and Automation, pp. 618-621, 2000.

[6] R. A. Beasley and R. D. Howe, "Tactile tracking of arteries in robotic surgery," IEEE Intl. Conf. on Robotics and Automation, Vol. 4, pp. 3801-3806, 2002.

[7] J. M. Hollerbach and C. W. Wampler, "The calibration index and taxonomy for robot kinematic calibration methods," Intl. J. of Robotics Research, vol. 15, no. 6, pp. 573-591, 1996.

[8] Z. Roth, B. W. Mooring, B. Ravani, "An overview of robot calibration," IEEE Trans. of Robotics and Automation, vol 3, no 5, pp 377-385, 1987.

[9] D. M. Rovner and R. H. Cannon, Jr., "Experiments toward on-line identification and control of a very flexible one-link manipulator," Intl. J. of Robotics Research, vol. 6, no. 4, pp. 3-19, 1987

[10] D.-S. Kwon and W. J. Book, "A time-domain inverse dynamic tracking control of a single-link flexible manipulator," J. of Dynamic Systems, Measurement, and Control, vol. 116, pp. 193-200, 1994.

[11] M. Jägersand, "Visual servoing using trust region methods and estimation of the full coupled visual-motor Jacobian" IASTED Applications of Robotics and Control, pp. 105-108, 1996.

[12] K. Hosoda and M. Asada, "Versatile visual servoing without knowledge of true Jacobian," Intl. Conf. on Intelligent Robots and Systems, pp. 186193, 1994

[13] C. C. Cheah, M. Hirano, S. Kawamura, and S. Arimoto, "Approximate Jacobian control for robots with uncertain kinematics and dynamics," IEEE Trans. Robotics and Automation, vol 19, no. 4, pp. 692-702, 2003. 